\title{
Finding small degree factors of lacunary polynomials
}

\author{
H.W. Lenstra, Jr.
}

\section{To Andrzej Schınzel}

\begin{abstract}
If $K$ is an algebraic number field of degree at most $m$ over the field $\mathbf{Q}$ of rational numbers, and $f \in K[X]$ is a polynomial with at most $k$ non-zero terms and with $f(0) \neq 0$, then for any positive integer $d$ the number of irreducible factors of $f$ in $K[X]$ of degiee at most $d$, counted with multiplicities, is bounded by a constant that depends only on $m, k$, and $d$ This is proved in a companion paper ( $\mathrm{H} W$ Lenstra, Jr "On the factorization of lacunary polynomials") In the piesent paper an algorithm for actually finding those factors is presented The algorithm assumes that $K$ is specified by means of an irreducible polynomial $h$ with integral coefficients and leading coefficient 1 , such that $K=\mathbf{Q}(\alpha)$ for a zero $\alpha$ of $h$ Also, the polynomial $f=\sum_{1} a_{2} X^{2}$ is supposed to be given in its sparse representation, $1 c$, as the list of pairs $\left(\imath, a_{i}\right)$ for which $a_{r} \neq 0$, each $\alpha_{r}$ being represented by means of its vector of coefficients on the vector space basis $1, \alpha$, , $\alpha^{(\mathrm{d} \operatorname{cg} h)-1}$ of $K$ over $\mathbf{Q}$ If $l$ denotes the "length" of these input data, when written out in binary, then the running time of the algorithm, measured in bit operations, is at most $(l+d)^{c}$ for some absolute and effectively compuiable constant $c$ Taking $K=\mathbf{Q}$ and $d=1$, one deduces that all rational zeroes of a sparsely represented polynomial with rational coefficients can be found in polynomial time This answers a question rased by F Cucker, P Konan, and S Smale
\end{abstract}

1991 Mathematıcs Subject Classification Primary 11R09, IIY16

Kcy words lacunary polynomial, computational complexity

Acknowledgements. The author was supported by NSF under grant No DMS 92-24205 He thanks J A Carık, C J Smyth, and J D Vaaleı for helpful assistance

\section{Introduction}

F Cucker, P Koiran, and S Smale [2] exhibited a polynomial time algorithm accomplishing the following Suppose that a polynomial $f=\sum_{l} a_{1} X^{\imath}$ in one variablo with cocfficients in the ring $\mathbf{Z}$ of integers is specified in its sparse representation, $1 \mathrm{c}$, by the list of pairs $\left(\imath, a_{1}\right)$ for which $a_{\imath} \neq 0$ Then the algorithm finds all zorocs of $f$ in $\mathbf{Z}$ One of the questions they ralsed is whether one can also find all rational seroes of $f$ in polynomial time In the present paper I show that this is indeed the 
case Rational zeroes correspond to irreducible factors of degree 1 over the field $\mathbf{Q}$ of rational numbers, and my result extends to finding irreducible factors of low degrees over algebraic number ficlds

For a ring $R$, let $R[X]$ denote the ring of polynomials in one variable $X$ over $R$ A polynomial is monuc if its leading coefficient is 1

Theorem. There is a deterministıc algorithm that, for some positıve rcal number $c$, has the following property given an algebran number field $K$, a sparsely represented non-zero polynomial $f \in K[X]$, and a posituve unteger $d$, the algorithm finds all monic urredurible factors of $f$ in $K[X]$ of degree at most $d$, as well as thear multiplicities, and it spends time at most $(l+d)^{c}$, where $l$ denotes the length of the input data

The conventions in this theorem are as in [8, Section 2] Rational numbers are represented as fractions of integers An algebraic number ficld $K$ is supposed to be specified by means of a monic irreducible polynomial $h \in \mathrm{Z}[Y]$ such that $K=\mathrm{Q}(\alpha)$ for a zero $\alpha$ of $h$, an clement of $K$, such as a coefficient of $f$, is then represented by means of its vector of coefficients on the vector space basis $\left(\alpha^{j}\right)_{j=0}^{m-1}$ of $K$ over $\mathbf{Q}$, where $m=\operatorname{deg} h$ Here the polynomial $h=\sum_{\jmath=0}^{m} h, Y^{\jmath}$ is densely represented, $1 \mathrm{e}$, by means of the list of all pars $(\jmath, h),, 0 \leq \jmath \leq m$, including those for which $h_{1}=0$ The length (or the szze) of the input data is defined in $[8,21]$ (ct [2, Sec 1]), it may informally be thought of as the number of bits necded to spell out the data in binary The time taken by an algorithm is measured in bit operations

One way of finding the irreducible factors of $f$ in $K[X]$ is first to convert $f$ from its sparse to its dense representation and next to apply one of the wellknown polynomal time algorithms (sec [1, 6]) for factoring densely represented polynomials over algebraic number fields This procedure, however, falls to satisfy the time bound stated in the theorem Consider, for example, the case in which $f=X^{n}-1$ for large $n$, with fixed $d$ and $K$, then the length $l$ of the data has order of magnitude $\log n$, and the length of the dense representation of $f$, which is about $n$, is exponential in $l$, so it cannot be written down within timc $(l+d)^{c}$

Our result is "uniform in $K$ " rather than having a scparate algorithm for cach $K$, we have one single algorithm that accepts data specifyng $K$ as part of the input, for fixed $d$, the running time is polynomially bounded in terms of the length of these datd and the datd specifyng $f$ For varying $d$, the running time can still be sard to be polynomally bounded in terms of the length of the input data and the possible length of the output, since the polynomals produced by the algorithm are densely represented and may have degree up to $d$ However, the algorithm may spend time exponential in $\log d$ and still find no factors

The number of different factors found by the algorithm is at most an absolute constant times $k^{2} 2^{n} n \log (2 n k)$, where $k$ is the number of non-zero terms of $f$ and $n=d\left[\begin{array}{ll}K & \mathbf{Q}\end{array}\right]$, by [9, Theorem 1] This is an exponential bound, but it is completely independent of the degree and the coefficients of $f$ and of the coefficients of the polynomal defining $K$ 
The idea behind the algorithm is best illustrated on an easier problem. Suppose that a sparsely represented polynomial $f \in \mathbf{Q}[X]$ as well as a rational number $x$ are given. How does one test in polynomial time whether or not $f(x)$ vanishes? Just substituting $x$ for $X$ in $f$ is not feasible, since if the degree of $f$ is very large then $f(x)$ may be too large to write down, let alone to calculate. Fortunately, if it is just a matter of testing whether $f(x)$ vanishos, one can get away with a much simpler procedure. Namely, excluding the easy cases $x= \pm 1$, one proves that a large degree polynomial with not many non-zero terms can vanish in $x$ only if it does so for obvious reasons, namely if there are "widely" spaced non-negative integers $u$ and "low" degree polynomials $f_{u}$ with $f_{u}(x)=0$ and $f=\sum_{u} f_{u} \cdot X^{u}$. The bounds that make this statement valid depend on the number of non-zero terms of $f$ and on the sizes of the numerators and denominators of its coefficients, but they do not depend on $x$. Thus, to test whether $f$ vanishes at a given rational number $x \neq \pm 1$, one "breaks" $f$ into appropriate polynomials $f_{\imath}$ and one tests whether thcy all vanish at $x$.

The algorithm underlying our theorem follows the same idea, and it is presented in Section 4. The basic result justifying the procedure (Proposition 2.3) is formulated and proved in Section 2. Section 3 contains several auxiliary algorithms, one of which finds the cyclotomic factors of $f$. The phenomenon that these require separate treatment is familiar from Schinzel's work on factors of lacunary polynomials.

Should the need for finding small degree factors of sparse polynomials over algebraic number ficlds cver arise, then a suitable variant of my method may very well have practical value; however, as it stands it is designed only to lead to a valid and efficient proof of the theorem.

Several results in this paper assert the existence of algorithms with certain properties. In each case, such an algorithm is actually exhibited in the paper itsclf or in one of the references. All these algoritbms are deterministic, and the constants appearing in running time estimates are effectively computable. Polynomials are densely represented in algorithms, unless it is explicitly stated that they are sparsely represented.

By $\mathbf{R}$ we denote the field of real numbers, and by $\mathbf{C}$ the ficld of complex numbers. The degree of a field extension $E \subset F$ is written $[F: E]$. The multiplicative group of non-zero elements of a field $F$ is denoted by $F^{*}$.

\section{Heights and lacunary polynomials}

Let $\overrightarrow{\mathbf{Q}}$ denote an algebraic closure of $\mathbf{Q}$, and let $K \subset \overline{\mathbf{Q}}$ be a finite extension of $\mathbf{Q}$. Write $M_{K}$ for the set of non-trivial prime divisors of $K$, and for each $v \in M_{K}$, let $\|\cdot\|_{v}: K \rightarrow \mathbf{R}$ be a corresponding valuation; we assume that these valuations are normalized as in [5, Chap. 2, Scc. 2]. This normalization is characterized by the facts that the product formula

$$
\prod_{v \in M_{K}}\|x\|_{v}=1 \quad \text { for all } x \in K^{*}
$$


holds, and that the relative herght function

$$
H_{K} \quad K \rightarrow \mathbf{R}, \quad H_{K}(x)=\prod_{v \in M_{K}} \max \left\{1,\|x\|_{v}\right\}
$$

(see $\left[5\right.$, Chap 3 , Sec 1]) satisfies $H_{K}(k)=k^{[K \mathrm{Q}]}$ for all positive integers $k$

The absolute herght function $H \quad \mathbf{Q} \rightarrow \mathbf{R}$ is defined by

$$
H(x)=H_{K}(x)^{1 /[K \mathbf{Q}]},
$$

where $K$ is such that $x \in K$, this is independent of the choice of $K$ For example, for $r, s \in \mathbf{Z}, s>0, \operatorname{gcd}(r, s)=1$ one has $H(r / s)=\max \{|r|, s\}$

For a positive integer $n$, we define

$$
c(n)=\frac{2}{n(\log (3 n))^{3}} \quad \text { if } n \geq 2,
$$

and $c(1)=\log 2$ This is a decrcasing function of $n$

Proposition 2.2. Let $n$ be a positive integer Suppose that $x \in \overline{\mathbf{Q}}^{*}$ us of degree at most $n$ over $\mathbf{Q}$, and that $\log I I(x)<c(n)$ Then $x$ us a root of unity

Proof See [12, Corollary 2] This proves 22

If $K$ is as above, then for $v \in M_{K}$ we extend \|\|$_{0}$ to a function $K[X] \rightarrow \mathbf{R}$ by $\left\|\sum a_{l} X^{2}\right\|_{v}=\max ,\left\|a_{\iota}\right\|_{v}$, Define $\mathbf{H} \quad \overline{\mathbf{Q}}[X] \rightarrow \mathbf{R}$ by $\mathbf{H}(f)=\prod_{v \in M_{K}}\|f\|_{\vartheta}^{1 /[K \mathbf{Q}]}$, where $K$ is chosen such that $f \in K[X]$, this is independent of the chorce

Proposition 2.3. Let $k, t, u$ be non-negative integers, and let $f \in \overline{\mathbf{Q}}[X]$ be a polynomal with at most $k+1$ non-zcro terms Suppose that $n$ is a positue integer with

$$
u-t>\frac{\log (k \mathbf{H}(f))}{c(n)},
$$

and that $f$ is wrattcn as the sum of two polynomials $g, h \in L[X]$ such that every non-zcro tcrm of $g$ has degree at most $t$ and every non-zero term of h has degree at least $u$ Then everyzcro of $f$ in $\mathbf{Q}^{+}$that has degrec at most $n$ over $\mathbf{Q}$ and that is not a root of unity is a common zero of $g$ and $h$

Proof Let $x \in \mathbf{Q}^{+}$be of degree at most $n$ over $\mathbf{Q}$, and suppose that $f(x)=0$ Then we have $g(x)=-h(x)$ We shall assume that $g(x)=-h(x) \neq 0$, and prove that $x$ is a root of unity

Let $K$ be chosen such that $x \in K$ and $f \in K[X]$ Then we have $g, h \in K[X]$ Let $v \in M_{K}$ From $h(x) \neq 0$ it follows that $h$ has at least 1 non-zero term, and since $f$ has at most $k+1$ non-zero terms 11 follows that $q$ has at most $k$ non-zero terms Thus $g(x)$ is a sum of at most $k$ terms $a, x^{\prime}$, with $\|a,\|_{0} \leq\|f\|_{0}$ and $\iota \leq t$ This leads to the estimate

$$
\|g(r)\|_{1} \leq \max \left\{1, \| k_{1}\right\} \quad\|f\|_{1},\|x\|_{v}^{\prime} \quad \text { if }\|x\|_{2} \geq 1
$$


Likewise, $h(x)$ is a sum of at most $k$ terms $a_{*} x^{i}$, with $\left\|a_{i}\right\|_{u} \leq\|f\|_{v}$ and $i \geq u$, so

$$
\|h(x)\|_{v} \leq \max \left\{1,\|k\|_{v}\right\} \cdot\|f\|_{v} \cdot\|x\|_{v}^{u} \quad \text { if }\|x\|_{v} \leq 1 .
$$

We have $\|g(x)\|_{v}=\|h(x)\|_{0}$, so we can combine these two statements in

$$
\max \left\{1,\|x\|_{v}\right\}^{u-\imath} \cdot\|g(x)\|_{v} \leq \max \left\{1, \| k_{v}\right\} \cdot\|f\|_{v} \cdot\|x\|_{v}^{u} .
$$

Raise this to the power $1 /[K: \mathbf{Q}]$ and take the product over $v \in M_{K}$. Using the tact that $H(k)=k$, and applying (2.1) to $x$ and to $g(x)$ (which are both supposed to be non-zero), one finds that

$$
H(x)^{u-\ell} \leq k \cdot \mathbf{H}(f) .
$$

By hypothesis, we have $k \cdot \mathbf{H}(f)<\exp ((u-t) c(n))$. It follows that $\log H(x)<c(n)$, so 2.2 implies that $x$ is a root of unity. This proves 2.3 .

Proposition 2.4. Let $K \subset \overline{\mathbf{Q}}$ be a finute extension of $\mathbf{Q}$, and let $f \in K[X]$. Let $r$ be a positive integer such that all coefficrents of $r f$ are algebranc untegers, and let $s$ be a positive real number with the property that for every field homomorphism $\sigma: K \rightarrow \mathbf{C}$ and every coefficient a of $f$ one has $|\sigma a| \leq s$. Then one has $\mathbf{H}(f) \leq r s$.

Proof. First assume that $r=1$. Then each cocfficient of $f$ is an algebraic integer, so $\|f\|_{v} \leq 1$ for each non-archimedean $v \in M_{K}$. Also, by definition of $s$ we have $\|f\|_{v} \leq s$ for each real $v \in M_{K}$, and $\|f\|_{v} \leq s^{2}$ for cach complex $v \in M_{K}$. Collecting all $v$, one obtains $\mathbf{H}(f) \leq s$, since the number of real $v$ plus twice the number of complex $v$ equals $[K: \mathbf{Q}]$. The case $r>1$ is reduced to the case $r=1$ by the formula $\mathbf{H}(r f)=\mathbf{H}(f)$, which follows from (2.1), applied to $x=r$. This proves 2.4 .

\section{Auxiliary algorithms}

Proposition 3.1. There us an algorithm that, for some positive constant $c_{1}$, has the following property: given an algcbrazc number field $K$ and a densely ropresented non-zero polynomial $f \in K[X]$, the algorthm finds the complete factorization of $f$ into monic urreducuble factors in $K[X]$, and it does so in time at most ${ }^{\wedge}$, where $l$ denotes the length of the data.

For the proof of this proposition, and a description of the algorithm, we refer to $[4 ; 6]$. It makes use of lattice basis reduction [7].

Let $K$ be a field of characteristic zero. For $f \in K[X]$, we define the sparse dervative $f^{[1]}$ of $f$ to be the ordinary derivative of $f / X^{\prime}$, if $X^{\prime}$ is the highest power of $X$ dividing $f$, and we define it to be 0 if $f=0$; the higher sparse derivatives $f^{[l]}$ are defined inductively by $f^{[l]}=\left(f^{[1-1]}\right)^{[1]}$, and for convenience we set $f^{[0]}=f$. If $f \neq 0$, then clearly the number of non-zcro terms of $f^{[1]}$ is one less than the number of non-zero terms of $f$. It follows that $f^{[\imath]}=0$ if and only if $\imath$ is greater than or equal to the number of non-zcro terms of $f$. 
Proposition 3.2. Let $K$ be a field of characterstuc zero, let $f \in K[X]$ be a nonzero polynomial, and let $g \in K[X]$ be an urrcducıble polynomial with $g(0) \neq 0$ Then the number of factors of $g$ in $f$ is equal to $\min \left\{\imath \geq 0 \quad g\right.$ does not divide $\left.f^{[\imath]}\right\}$, and it us smaller than the number of non zero terms of $f$

Proof The first assertion is proved in a routine manner by induction on the number of factors of $g$ in $f$ If $f$ has exactly $k+1$ terms, then $f^{[k]}$ is a polynomial with exactly one term, which is not divisible by $g$ Thus the sccond assertion follows from the first This proves 32

The second assertion can also be derived from an observation of Hajos (sce $[3,11$, Lemma 1])

Proposition 3.3. There us an algorithm that, for some positive constant $c_{2}$, has the following property Given an algebraic number field $K$ and a sparsely represented non-zrro polynomial $f \in K[X]$, the algorithm computes the sparse repre scntations of the sparse dervvatives $f^{[l]}$ for all $\imath \geq 0$ that are less than the number of non zero terms of $f$ and it does so in tume at most $l^{\prime 2}$, where $l$ denotes the lingth of the data

Proof This is obvious - one just computes the polynomials $f^{[2]}$ directly from the definition Thus proves 33

Proposition 3.4. There us an algornthm that, for some positive constant $c_{3}$, has the following propcrty given an algebraic number field $K$, a sparsely represented non yero polynomal $f \in K[X]$, and a positive intrger $r$, the algorithm computes the grfatest common divisor of $f$ and $X^{r}-1$ in $K[X]$, and tt docs so in time at most $(l+r)^{3}$, wherel dcnotes the length of the data

Proof The algorithm runs ds follows Let $f=\sum_{1} a, X^{t()}$ For cach ?, compute the remainder $u(\imath)$ of $t(\iota)$ upon division by $r$ Next compute the polynomial $h=\sum_{1} a_{1} X^{u(1)}$, and use the Euclidean algorithm for polynomials in order to compute the greatest common divisor of $h$ with $X^{r}-1$. This gcd is the output of the algorithm

To prove the correctncss, it suffices to remark that from $t(\imath) \equiv u(\imath) \bmod r$, for cach $\iota$, it follows that $f \equiv h \bmod X^{r}-1$, and therefore $\operatorname{gcd}\left(f, X^{r}-1\right)=$ $\operatorname{gcd}\left(h, X^{r}-1\right)$

The running time estmmate is proved in a completely straightforwald way, note that $h$ is densely represented, and has degree less than $r$ For a running time c stimate of the Euclidean algorithm for polynomials, see [4, Cor 18 ] This proves Proposition 34

If $K$ is a field, we call a polynomial $g \in K[X]$ cyclotomuc if, for some positive intcger $r$, it is a monic irreducible factor of $X^{\prime}-1$ in $K[X]$

Proposition 3.5. There 25 an algorthm that, for some positive constant ${ }_{4}$, has the following property gucn an algebranc number field $K$, a sparsely rcpresented 
non-zero polynomial $f \in K[X]$, and a positive integer $d$, the algorthm computes in time at most $(l+d)^{c_{4}}$ all cyclotomic factors $g$ of $f$ in $K[X]$ that have degree at most $d$, as well as, for each such $g$, the multuplicnty $m(g)$ of $g$ as a factor of $f$, here $l$ denotes the length of the input data

Proof Wo claim that the following algorithm has the stated properties It produces a list of pairs $g, m(g)$, which is initially supposed to be empty

For each integer $r=1,2, \quad, 2\left(d\left[\begin{array}{ll}K & \mathrm{Q}\end{array}\right]\right)^{2}$ in succession, do the following Compute $\operatorname{gcd}\left(f, X^{r}-1\right)$ with the algorithm of 34 , factor $\operatorname{gcd}\left(f, X^{r}-1\right)$ into irreducible factors in $K[X]$ by means of the algornthm of 31 , and discard those urreducible factors that appear already on the list or have degree greater than $d$ Adjoin the remaining irreducible factors $g$ to the list, and for each of them compute $m(g)$ trom the formula

$$
m(g)=\min \left\{z \quad 1 \leq \imath \leq k, g \text { does not divide } \operatorname{gcd}\left(f^{[\imath]}, X^{\gamma}-1\right)\right\},
$$

where $k$ is one less than the number of non-zero terms of $f$, here $f^{[q]}$ is computed in its sparse representation by the algorithm of 33 , and its gcd with $X^{\prime}-1$ is computed in its dense representation as in 34

This completes the description of the algorithm

The proof of the bound for the running time is straightforward, and left to the reader We prove that each cyclotomic factor $g$ of $f$ of degree at most $d$ is found by the algorithm, and that $m(g)$ is its multiplicity Let $g$ be such a factor, let $\zeta$ be a zero of $g$ in an extension field of $K$, and let $r$ be the multiplicative order of $\zeta$ Denoting the Euler $\varphi$-function by $\varphi$, we have

$$
\begin{aligned}
\varphi(r)=\left[\begin{array}{ll}
\mathbf{Q}(\zeta) & \mathbf{Q}
\end{array}\right] \leq\left[\begin{array}{ll}
K(\zeta) & \mathbf{Q}
\end{array}\right]=\left[\begin{array}{ll}
K(\zeta) & K
\end{array}\right]\left[\begin{array}{ll}
K & \mathbf{Q}
\end{array}\right] \\
=\left(\begin{array}{ll}
\operatorname{deg} g
\end{array}\right) \quad\left[\begin{array}{ll}
K & \mathbf{Q}
\end{array}\right] \leq d \quad\left[\begin{array}{ll}
K & \mathbf{Q}
\end{array}\right]
\end{aligned}
$$

The elementary inequality $\varphi(r) \geq \sqrt{r / 2}$ now implies that $r \leq 2\left(d \quad\left[\begin{array}{ll}K & \mathbf{Q}\end{array}\right]\right)^{2}$ Therefore $g$ is indecd found by the algorithm From Proposition 32 it follows that $m(g)$ equals the multiplicity of $g$ as a tactor of $f$ This proves 35

The function $\mathbf{H}$ in the following result is as defined in Section 2, with $\mathbf{Q}$ equal to an algebraic closure of $\mathbf{Q}$ that contains $K$

Proposition 3.6. There is an algonthm that, for some positive constant $c_{5}$, has the following property given an algebraic number field $K$ and a sparsely represented non zero polynomial $f \in K[X]$, the algsrithm computes in time at most $l^{c_{0}}$ a positive integer $b$ satisfying $b \geq k \quad \mathbf{H}(f)$, here $k$ is 1 less than the number of non-zero terms of $f$, and $l$ denotes the length of the input data

Proof As in the introduction, it is assumed that $K$ is specified by means of an irreducible polynomial $h=\sum_{j=0}^{m} h_{j} Y^{\prime} \in \mathbf{Z}[Y]$, with $h_{m}=1$, with the property that $K=\mathbf{Q}(\alpha)$ for some zero $\alpha$ of $h$ Also, each coefficient $a_{2}$ of $f$ is supposed to be represented by a vector $\left(q_{1,}\right)_{j=0}^{m-1}$ with $q_{2 \jmath} \in \mathbf{Q}$ for which $a_{1}=\sum_{\jmath=0}^{m-1} q_{1 \jmath} \alpha^{\jmath}$ For each field homomorphism $\sigma \quad K \rightarrow \mathbf{C}$, the complex number $\sigma \alpha$ is a $<$ ero of $h$ and therefore satisfies $|\sigma \alpha| \leq B=\sum_{j=0}^{m-1}\left|h_{\jmath}\right|$ Hence if $r$ is a positive integer for which 
$r \cdot q_{1,} \in \mathbf{Z}$ for all $i$ and $j$, then one has

$$
\left|\sigma\left(r \cdot a_{\imath}\right)\right| \leq s_{\imath}=\sum_{\jmath=0}^{m-1}\left|r \cdot q_{\eta}\right| \cdot B^{\jmath}
$$

for all field homomorphisms $\sigma: K \rightarrow \mathbf{C}$ and all $i$. Thus, by 2.4 the number $b=k \cdot \max _{1} s_{b}$ is a positive integer satisfying $b \geq k \cdot \mathbf{H}(f)$. One can computc $b$ in polynomial time in a straightforward way, taking for $r$ the least common multiple (or even the product) of the denominators of the $q_{\imath}$. This proves 3.6.

\section{Proof of the theorem}

The proof of the theorem stated in the introduction consists of three parts: the description of the algorithm underlying the theorem, the proof of its correctness, and the running time estimate.

To describe the algorithm, let an algebraic number field $K$, a sparsely represented non-zero polynomial $f \in K[X]$, and a positive integer $d$ be given. The algorithm produces a list of pairs $g, m(g)$, which is initially supposed to be empty.

Step 1. Find the cyclotomic factors. Use the algorithm of 3.5 to find all cyclotomic factors $g$ of $f$ in $K[X]$, as well as their multiplicities $m(g)$.

Step 2. Compute a bound for the gap wrdth. Let $k+1$ be the number of non-zero terms of $f$. Use the algorithm of 3.3 to compute $f^{[1]}$ for $0 \leq i<k$ in their sparse representations. Next, applying the algorithm of 3.6 to each $f^{[1]}$, compute positive integers $b_{\iota}$ satisfying

$$
b_{\imath} \geq(k-i) \cdot \mathbf{H}\left(f^{[\imath]}\right) \quad \text { for } i=0,1, \ldots, k-1 .
$$

Finally, compute a positive integer $b$ satisfying

$$
b \geq \frac{\max \left\{\log b_{1}: 0 \leq i<k\right\}}{c(d \cdot[K: \mathbf{Q}])}>b-2,
$$

with the function $c$ as defined in Section 2. For the logarithms, one can use the algorithms in [1]. (For the significance of $b-2$, see $[10$, Sec. 1, end].)

Step 3. Splat $f$ at the brg gaps. Let $f=\sum_{t \in T} a_{l} X^{t}$, where $T$ is a set of $k+1$ non-negative integers and $a_{t} \in K^{*}$ for each $t \in T$. Ordering $T$, determine the subset $U=\{u \in T$ : there does not exist $t \in T$ with $u-b \leq t<u\}$ of $T$, where $b$ is as computed in Step 2. Next, for each $u \in U$, dctermine the subset $T(u)=\{t \in T: u=\max \{v \in U: v \leq t\}\}$ of $T$. (Then $T$ is the disjoint union of the sets $T(u)$, for $u \in U$, and each $T(u)$ contains $u$.) To conclude this step, compute the polynomials

$$
f_{u}=\sum_{t \in T(u)} a_{1} X^{1-u} \quad(u \in U),
$$

in their dense representations. (These polynomials satisfy $f_{\varkappa}(0) \neq 0$ and $f=\sum_{u \in U} f_{u} \cdot X^{u}$.) 
Step 4. Factor a dense polynomial. Using the Euclidean algorithm for polynomials (see [4, Cor. 1.8]), compute $h=\operatorname{gcd}_{u \in U} f_{u}$. Factor $h$ into monic irreducible factors in $K[X]$ by means of the algorithm of 3.1 .

Step 5. Assemble the results. Discard each monic irreducible factor of $h$ that occurs already among the factors computed in Step 1 or has degree greater than $d$. Adjoin each of the remaining monic irreducible factors $g$ of $h$ to the list, with $m(g)$ cqual to the multiplicity of $g$ as a factor of $h$. Finally, if 0 does not belong to the set $T$ of Step 3, adjoin $g=X$ to the list, with $m(X)$ equal to the smallest element of $T$.

This concludes the description of the algorithm.

We noxt prove the correctness. The parenthetical statements in Step 3 are readily vorified. The polynomial $h$ divides each $f_{u}$, so it divides $f$. One deduces that the polynomials $g$ produced by the algorithm are indeed monic irroducible factors of $f$ in $K[X]$ of degree at most $d$. Also, $h$ is not divisible by $X$, since none of the $f_{u}$ is, so from Step 5 one sees that no $g$ is produced twice.

Conversely, let $g$ be a monic irreducible factor of $f$ in $K[X]$ of degree at most $d$. We prove that $g$ is produced by the algorithm, and that $m(g)$ equals the multiplicity of $g$ as a factor of $f$. These statements are obvious if $g$ is cyclotomic (Step 1 ) and if $g=X$ (Step 5). In the other case, let $\overline{\mathbf{Q}}$ be an algebraic closure of $\mathbf{Q}$ containing $K$, and let $x \in \overrightarrow{\mathbf{Q}}$ be a zero of $g$. By hypothesis, $x$ is not a root of unity, and $x \neq 0$. The degree $[\mathbf{Q}(x): \mathbf{Q}]$ of $x$ over $\mathbf{Q}$ satisfies

$$
[\mathbf{Q}(x): \mathbf{Q}] \leq[K(x): \mathbf{Q}]=[K(x): K] \cdot[K: \mathbf{Q}]=(\operatorname{deg} g) \cdot[K: \mathbf{Q}] \leq d \cdot[K: \mathbf{Q}] .
$$

For each $u \in U$, we now apply 2.3 with $n=d \cdot[K: \mathbf{Q}]$, and with

$$
\sum_{v \in U, v<u} f_{v} \cdot X^{v}, \quad \sum_{v \in U, v \geq u} f_{v} \cdot X^{v}
$$

in the roles of $g$ and $h$. From

$$
\frac{\log (k \cdot \mathbf{H}(f))}{c(n)} \leq \frac{\log b_{1}}{c(d \cdot[K: \mathbf{Q}])} \leq b
$$

and the definitions of $U$ and $f_{u}$ it follows that the inequality of 2.3 is satisfied. Now 2.3 asserts that $x$ is a zero of both polynomials just displayed. Since this is the case for each $u \in U$, one infers that $f_{u}(x)=0$ for all $u \in U$, and therefore that $h(x)=0$. Hence $g$ is an irreducible factor of $h$, and it is produced by the algorithm. To show that $m(g)$ is the multiplicity of $g$ in $f$, we repeat the argument just given with $f^{[l]}$ in the role of $f$, for each $i=1,2, \ldots, k-1$. The representation $f=\sum_{u \in U} f_{u} X^{u}$ induces a similar representation of cach $f^{[1]}$. Thanks to the choice of $b$ we can still apply (2.3). Using 3.2, one deduces that $x$ is a $j$-fold zero of $f$ if and only if it is a $j$-fold zero of each $f_{u}$, the case $j>k$ being vacuously correct. Thus, the multiplicity of $g$ as a factor of $f$ is the same as the multiplicity $m(g)$ of $g$ as a factor of $h=\operatorname{gcd}_{u} f_{u}$. This proves the correctness of the algorithm.

We prove the running time cstimate. Sincc each $b_{7}$, in Step 2, is computed by a polynomial time algorithm, its logarithm is bounded by a constant power of the length $l$ of the data. Also, from the definition of $c(n)$ in Section 2 one sces that 
$1 / c(n)$ is bounded by a constant times $n^{2}$ It follows that the bound $b$ computed in Step 2 is bounded by a constant power of $l+d$ Now let $u \in U$ The definitions of $U$ and $T(u)$ imply that any two consecutive non-zero terms of $f_{u}$ have degrees duffering by at most $b$ Since $f_{u}$ has at most $k+1$ non-zero terms, one of which has degrec 0 , it tollows that $\operatorname{deg} f_{u} \leq k \quad b$ Therefore the length of the dense representation of $f_{u}$ is bounded by a constant power of $l+d$ This implies that the time taken by the polynomial time operations on the $f_{u}$ in Step 4 remains within the bound stated in the theorem It is a routme matter to prove that this also applies to the time taken by the other steps of the algorithm

This proves the theorem stated in the introduction

\section{References}

[1] Brent, R P , Fdst multiple-precision evaluation of elementary functions J Assoc Comput Mach 23 (1976), 242-251

[2] Cucker, F, Koiran, P, Smale, S, A polynomal time algorithm for diophantıne equations in one variable $J$ Symbolic Comput, to appear

[3] Hajos, G, [Solution to problem 41] (in Hungarian) Mat Lapok 4 (1953), 4041

[4] Landau, S, Factormg polynomials over algebraic number fields SIAM J Comput 14 (1985), 184195

[5] Lang, S, Fundamentals of diophantme geometry Spinger, New York 1983

[6] Lenstra, A K, Factorng polynomals over algebraic number fields In Computer algebra (ed by J A van Hulıen, Lecture Notes in Comput Scı 162), 245-254 Springer, Berlın 1983

[7] Lenstra, A K, Lenstra, H W , Jr, Lovasz, L, Factoring polynomials with rational coefficients Math Ann 261 (1982), 515534

[8] Lenstra, H W, Ir, Algorithms in algebraic number theory Bull Amer Math Soc (N S) 26 (1992), 211-244

[9] - On the factorization of lacunary polynomials This volume, 277291

[10] Lenstrd, HW, Jr, Pomerance, C, A rigorous time bound for factoring integers J Amer Math Soc 5 (1992), 483516

[11] Montgomery, H L, Schnzel, A, Some arithmetic properties of polynomials in several variables In Transcendence theory advances and applications (ed by A Baker, DW Masser), Chapter 13, 195203 Academic Press, London 1977

[12] Voutier, P, An effective lower bound for the height of algebrarc numbers Acta Arth 74 (1996), 8195 